\title{
NEURAL NETWORKS BASED IDENTIFICATION OF HELICOPTER DYNAMICS USING FLIGHT DATA
}

\author{
S. Suresh ${ }^{1}$, M. Vijaya Kumar ${ }^{2}$, S. N. Omkar ${ }^{1}$, V. Mani ${ }^{1}$, Prasad Sampath ${ }^{2}$ \\ ${ }^{1}$ Aerospace Engineering Department, \\ Indian Institute of Science, Bangalore - 560012 \\ ${ }^{2}$ Rotary Wing Research and Design Center, \\ HAL, Bangalore - 560017.
}

\begin{abstract}
In this paper, identification of lateral and longitudinal dynamics of helicopter using flight data is presented. Neural networks with linear filter also known as Narendra's model and recurrent neural networks with internal memory (Memory Neuron Networks) are used for the purpose. These networks are used for identifying the rate and attitude response of the helicopter to a given longitudinal cyclic and lateral cyclic inputs. The relative effectiveness of these algorithms has been discussed.
\end{abstract}

\section{INTRODUCTION}

System identification in control engineering is a key element for understanding and controlling unknown nonlinear dynamical systems. Traditional System identification techniques such as least square estimation [1], quasi-linearisation [2] and stochastic modeling [3] have been successfully used in nonlinear dynamical systems. In traditional system, model structure must be defined a priori to estimate all required system parameters. In case of helicopter dynamics, defining a priori model is difficult because of highly nonlinear aeromechanical characteristics due to the interplay between various subsystems like rotor, fuselage, power plant, transmission, empennage, and tail rotor.

The linearised mathematical model is validated from the knowledge of control inputs and system response via flight test data collection. This analysis will improve the confidence and reduce the uncertainty in linearised model describing helicopter dynamics [11]. It is important to determine the lowest order linear model that would best fit the flight test data. Identifying the lowest linear model is difficult and time consuming [11]. Given input and output data, Artificial Neural Networks (ANN) is capable of identifying underlying relationship between the input and output data. Since the helicopter dynamic response is nonlinear, dynamical neural networks can be used $[4,5]$. The objective of this work is to identify the longitudinal and lateral dynamics of helicopter using dynamical neural network with linear filter and memory neuron networks [6-8].

Recently it is shown in [9] that models can be constructed using ANN and linear filters in cascade and/or feed back configurations for identifying nonlinear dynamical systems. This approach is easy to implement. But, one needs a fairly good knowledge of the structure of the system to decide suitable combination of memory-less transformations and linear filters can approximate the longitudinal dynamics. The requirements of large number of tapped delay lines in the network and the unknown order of the system make the use of above model difficult. This difficulty can be overcome by using recurrent networks with internal memory.

Recurrent networks with internally trainable temporal memory are called Memory Neuron Networks (MNN) [10]. They can be trained to model dynamical systems without explicitly feeding past inputs and outputs. MNN do not require any knowledge about the order of the system. The primary aim of this paper is to analyze different neural network models in terms of modeling accuracy and computational complexity for identification of helicopter dynamics. For this purpose two network models, namely multilayer feed forward networks with linear filters as described in [9], and MNN are chosen.

The paper is organized as follows; section 2 gives brief description about neural network models. Section 3 details about acquiring flight data at different conditions and simulation. The results are discussed in Section 4

\section{NETWORK MODELS}

In this section the different neural network models with corresponding learning algorithms chosen are briefly discussed. 
2.1 Multi layer feed forward networks with linear filters:

The network chosen here is similar to that proposed in [9]. The structure is that of a feed forward Artificial Neural Network (ANN) with Tapped Delay Lines (TDL). In this case the output of the plant can be described by,

$y_{p}(k+1)=f\left(u(k), u(k-1), . ., y_{p}(k), y_{p}(k-1), ..\right)-(1)$

A series-parallel model [9] is used for identification. The network output is then given by

$$
\hat{y}_{p}(k+1)=N\left(u(k), u(k-1), \dot{y}_{p}(k), y_{p}(k-1), . .\right)
$$

where, $\mathrm{N}$ is the network approximation function. As can be seen the order of the system should be known $a$ prioni for the purpose of identification. The network model is trained using BPTT as described in [9].

\subsection{Memory Neuron Network (MNN):}

The architecture of MNN is similar to the one proposed in [10]. The structure is same as feed forward ANN except for the memory neurons. At each level of the network except the output level, each of the network neurons has exactly one memory neuron connected to it [10]. The memory neuron takes input from the corresponding network neuron and it also-has self-feedback. This leads to storage of past values of the network neuron in the memory neuron. All the network neurons and the memory neurons send their output to the network neuron in the next layer. In the output layer, each network neuron can have cascade of memory neurons and each of them can send output to the network neuron in the output layer. The MNN is trained using BPTT as described in [10]:

\section{SIMULATION}

In this section, we briefly discuss about flight data acquisition and training and testing of dynamical neural networks.

\subsection{Acquisition of flight data:}

Flight test is carried out on a four bladed helicopter with conventional mechanical controls. Test is carried out in calm wind conditions. The helicopter is instrumented to measure pitch $(q)$, roll $(p)$ and yaw (r) rates, normal, lateral and longitudinal $\left(a_{z}, a_{y}, a_{x}\right)$ accelerations, pitch and roll attitude $(\theta, \phi)$, forward speed $(\bar{v})$, barometric altitude $(\mathrm{h})$ and side slip $(\beta)$. The four-control displacements namely, longitudinal cyclic $\left(D_{\beta}\right)$, lateral cyclic $\left(D_{\alpha}\right)$, collective $\left(D_{\theta}\right)$ and pedal input $\left(D_{\delta}\right)$ are also measured. The signals are sampled at $26 \mathrm{~Hz}$ for $6 \mathrm{sec}$ duration and stored in a on-board flight data recorder.

After trimming the helicopter, training data are generated by giving $+5 \%$ and $-5 \%$ of longitudinal cyclic input $\left(\mathrm{D}_{\beta}\right)$, at straight and level flight. The helicopter response is recorded for $6 \mathrm{sec}$. Similarly, for lateral inputs $(+5 \%$ and $-5 \%)$ the helicopter response is recorded for $6 \mathrm{sec}$ duration. These data are preprocessed for consistency check and stored. For validation purpose, the response of the helicopter for doublet input (for $D_{\beta}$ and $D_{\alpha}$ ) is measured. These data are normalized between \pm 0.8 in order "to train/validate the neural network.

\subsection{Training the neural network}

Longitudinal cyclic input $\left(D_{\beta}\right)$ and the corresponding primary responses pitch rate $(q)$ and pitch attitude $(\theta)$ are used to train the network $\mathrm{N}_{1}$. Similarly, lateral cyclic input $\left(D_{\alpha}\right)$ and corresponding primary responses roll rate $(p)$ and roll attitude $(\phi)$ are used to train the network $\mathrm{N}_{2}$.

\begin{tabular}{|c|c|c|c|c|}
\hline \multirow{2}{*}{$\begin{array}{c}\text { Network } \\
\text { parameters }\end{array}$} & \multicolumn{2}{|c|}{$\begin{array}{c}\text { Longitudinal } \\
\text { Dynamics }\end{array}$} & \multicolumn{2}{c|}{ Lateral Dynamics } \\
\cline { 2 - 5 } & Narendra & MNN & Narendra & MNN \\
\hline $\begin{array}{c}\text { No. of } \\
\text { input node }\end{array}$ & $\begin{array}{c}11(1+2 \\
+4 * 2)\end{array}$ & $\begin{array}{c}3(1+ \\
1 * 2)\end{array}$ & $\begin{array}{c}11(1+2 \\
+4 * 2)\end{array}$ & $\begin{array}{c}3(1+ \\
1 * 2)\end{array}$ \\
\hline $\begin{array}{c}\text { - No. of } \\
\text { output } \\
\text { node }\end{array}$ & $2 \cdots$ & 2 & 2 & 2 \\
\hline $\begin{array}{c}\text { No. of } \\
\text { hidden } \\
\text { layer }\end{array}$ & 1 & 1 & 1 & 1 \\
\hline $\begin{array}{c}\text { No. of } \\
\text { hidden } \\
\text { nodes }\end{array}$ & 20 & 10 & 20 & 10 \\
\hline $\begin{array}{c}\text { No. of } \\
\text { memory } \\
\text { neuron in } \\
\text { output } \\
\text { node }\end{array}$ & - & 2 & - & 2 \\
\hline Epoch & 1000 & 1000 & 1000 & 1000 \\
\hline $\begin{array}{c}\text { Learning } \\
\text { rate }\end{array}$ & 0.01 & 0.03 & 0.01 & 0.03 \\
\hline
\end{tabular}

\subsubsection{MLP with linear filter (Narendra model)}

In case of neural network with linear filters, apart from the actual inputs two past input and four past outputs are fed explicitly as input to the network $\mathrm{N}_{1}$ 
and $\mathrm{N}_{2}$ (assuming a fourth order system). The network parameters for both the networks are shown in the Table 1. The networks are trained and tested with different sets of data and the corresponding results are shown in figure 1-2.

\subsubsection{MNN}

Since MNN is capable of capturing the history dependency, both the networks $\mathrm{N} 1$ and $\mathrm{N} 2$ have one input and two outputs. Serial - parallel model is used in both the network and hence the effective input becomes three (actual input + one past outputs). The network parameters are listed in Table 1. The results of the training/testing are shown in figure 3-4.

\begin{tabular}{|c|c|c|c|c|}
\hline & \multicolumn{2}{|c|}{ Longitudinal $\left(\mathrm{N}_{1}\right)$} & \multicolumn{2}{c|}{ Lateral $\left(\mathrm{N}_{2}\right)$} \\
\cline { 2 - 5 } & Narendra & $\mathrm{MNN}$ & Narendra & $\mathrm{MNN}$ \\
\hline $\begin{array}{c}\text { Training } \\
\text { time }\end{array}$ & $\cong 10 \mathrm{sec}$ & $\cong 5 \mathrm{sec}$ & $\cong 11 \mathrm{sec}$ & $\cong 6 \mathrm{sec}$ \\
\hline $\begin{array}{c}\text { No. of } \\
\text { weights }\end{array}$ & 260 & 117 & 260 & 117 \\
\hline $\begin{array}{c}\text { Training } \\
\text { error }\end{array}$ & 0.7365 & 0.7285 & 0.6934 & 0.6999 \\
\hline $\begin{array}{c}\text { Testing } \\
\text { error }\end{array}$ & 0.8012 & 0.7912 & 0.8375 & 0.8175 \\
\hline
\end{tabular}

\section{RESULTS AND DISCUSSION}

Figure 1 and 2 show the identification of the rate and attitude response of the helicopter to a given longitudinal and lateral cyclic inputs for Narendra's model. The same identification problem carried out using MNN is shown in figure 3 and 4 . It can be easily seen from the above figures 3 and 4 , the results obtained are comparable to the result obtained using the algorithm discussed in [9]. In Table 2 the other important aspects such as total training time, no. of weights, training error and testing error for each networks are presented. From this table, we can see that the training and testing error for $\mathrm{MNN}$ is less than the Narendra's model because we assumed a fourth order system in Narendra's model. MNN approach takes less training time than the Narendra's model because the Narendra's model has more number of weights due to tapped delay lines. Number of weights used by both the networks is also shown in Table 2. Hence, it is advantageous to use the MNN approach for identification.

\section{CONCLUSION}

In this study, the identification problem of longitudinal and lateral dynamics of the helicopter is presented using Narendra's model and MNN. The results obtained using MNN approach are compared with Narendra's model. From the simulation studies it is shown that MNN approach is better than Narendra's approach. Fine-tuning of the network parameters can further enhance the performance of the networks.

\section{REFERENCES}

[1] K.J. Astrom and P.E. Eykhoff, "System Identification- A Survey", Automatica, Vol.7, pp. 123-162, 1971.

[2] R.Kalaba and K.Spingarn, "Control, Identification and Input Optimization”, Plenum press, 1982.

[3] B. Kuzta, "Modeling and identification of dynamic systems", Van Nostrand Reinhold Company, 1983.

[4] C Zhu and F.W. Paul, "A Fourier series neural network and its application to system identification", ASME Trans., Journal of dynamics systems, Measurement and control. Vol. 117, no. 3, pp. 253261, 1995.

[5]. W.T. Miller, R.S. Sutton and P.J. Werbose, "Neural networks for controls", Cambridge, MA, MIT press, 1990.

[6] L.L. Jung, "Issue in system identification", IEEE Control systems, vol. 11, no.1, pp. 25-29, 1991.

[7]Y. Ichikawa and T. Sawa, "Neural network application for direct regulator controllers", IEEE Trans. On Neural Networks, vol. 3, no. 2, pp. 224231, 1992.

[8] Q.H. Wu, B.W.Hogg and G.W. Irwin, "Neural network regulator for turbo generators", IEEE Trans. On Neural Networks, vol. 3, no. 1, pp. 95-100, 1992.

[9] K.S.Narendra and K.Parthasarathy, “ Identification and control of dynamical system using neural networks", IEEE Trans. on neural networks, vol.1, No.1, pp.4-27, 1990.

[10] P.S.Sastry, G.Santharam and K.P. Unnikrishnan, "Memory neuron networks for identification and control of dynamical systems", IEEE Trans. on neural networks, vol5, No.2, pp. 306-319, 1994.

[11] "Rotorcraft System Identification", AGARDAR-280, Sep, 1991. 

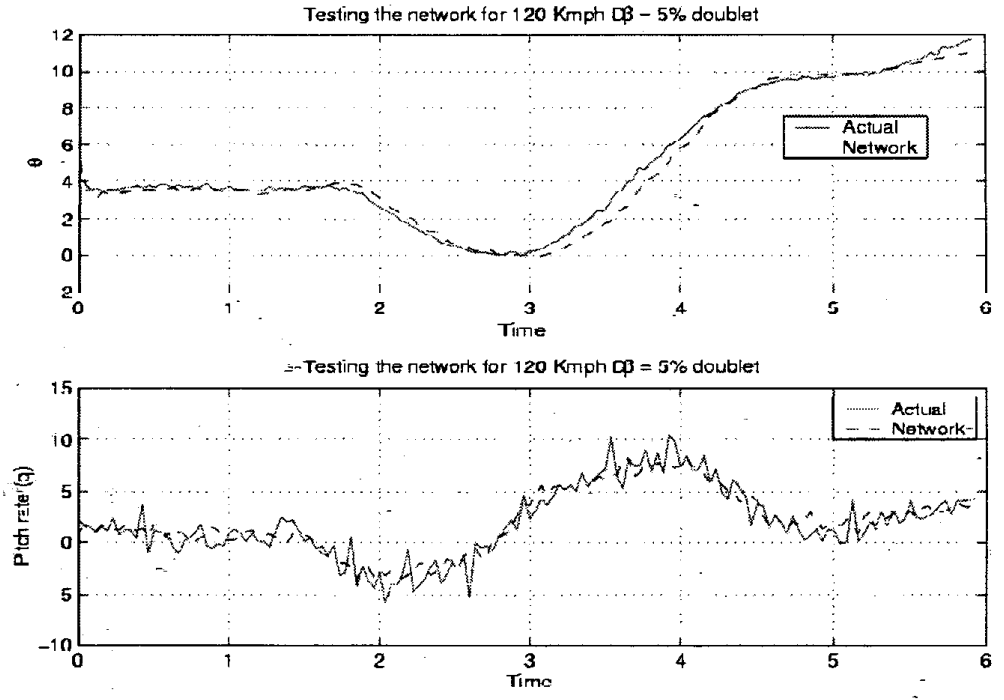

Figure 1. Testing the Narendra model $\left(\mathrm{N}_{1}\right)$ for $5 \%$ doublet input (Longitudinal response).
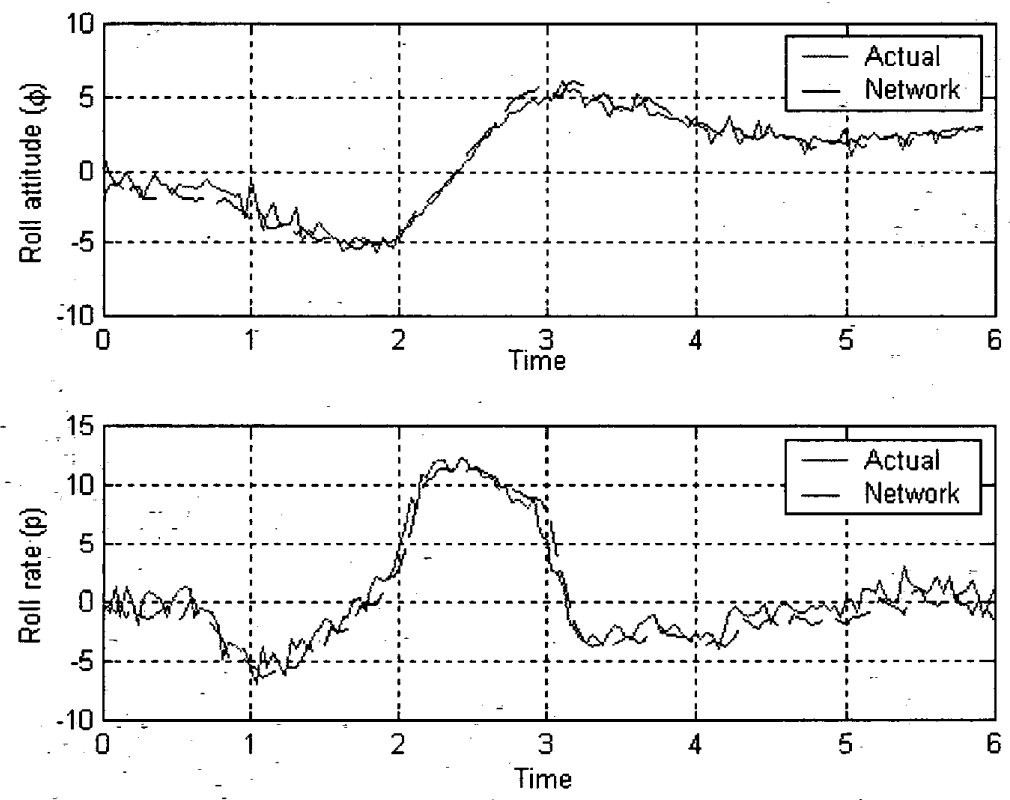

Figure 2. Testing the Narendra model $\left(\mathrm{N}_{2}\right)$ for $5 \%$ doublet (Lateral response). 

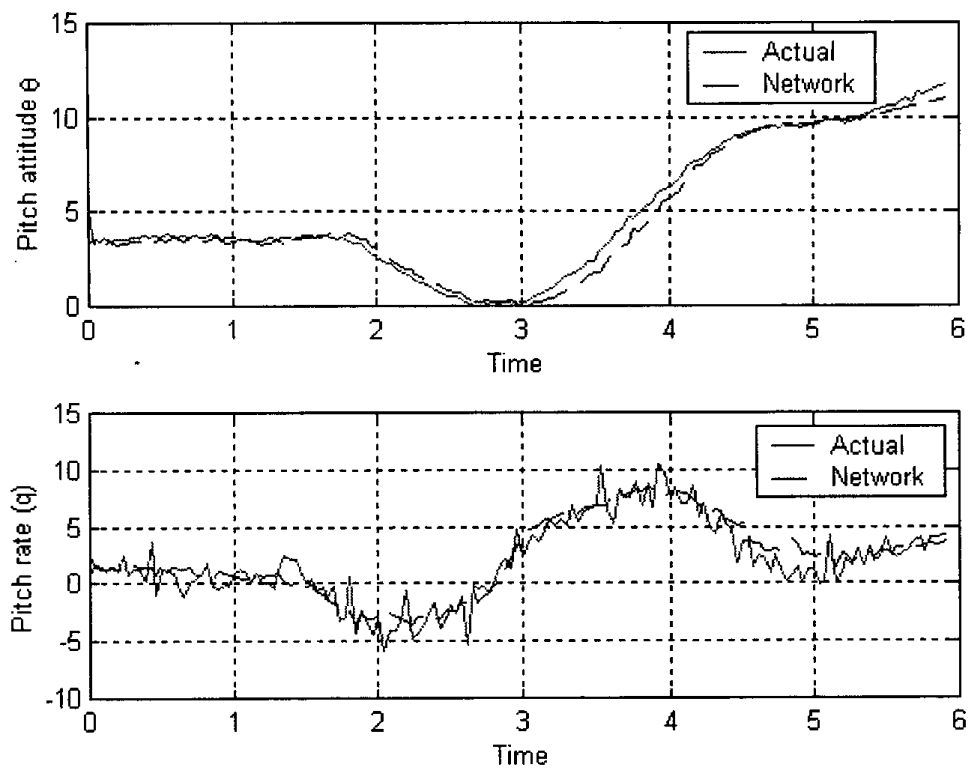

Figure 3. Testing the $\mathrm{MNN}$ model $\left(\mathrm{N}_{1}\right)$ for $5 \%$ doublet (Longitudinal response)
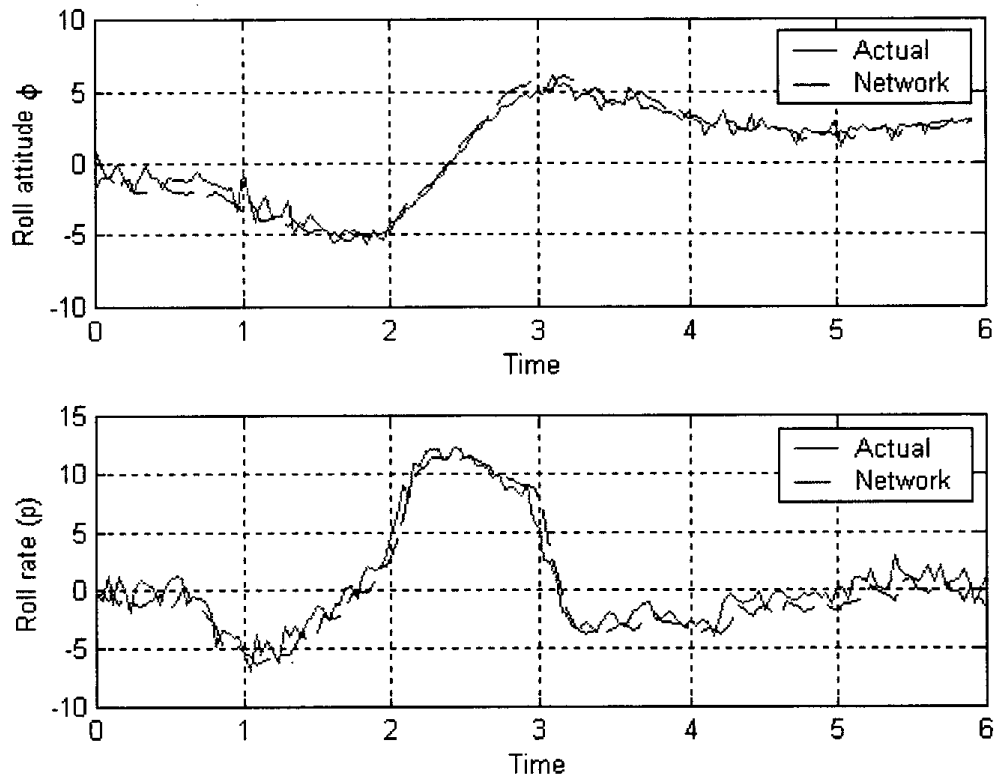

Figure 4. Testing the $\mathrm{MNN}$ model $\left(\mathrm{N}_{2}\right)$ for $5 \%$ doublet (Lateral response). 